\title{
Real time fire front monitoring through smoke with bi-spectral infrared imaging
}

\author{
J. M. Aranda, J. Meléndez, L. Chávarri \& F. López \\ LIR - UC3M: Laboratorio de Sensores Teledetección e Imagen IR \\ Departamento de Física, Universidad Carlos III de Madrid, Spain
}

\begin{abstract}
Infrared (IR) imaging is a standard technique in forest fire detection. In previous works we have shown that it can be used to classify the fire scene into regions (of embers, flame, fire front, etc). However, this requires multi-spectral images and a complex post-processing. In this paper we show that a less precise but still powerful classification of fire scenes, for distances in the range of hundreds of meters, can be done with a much simpler procedure. A fire index is obtained from bi-spectral images in the medium IR with an extremely simple processing that can be performed in real time. This makes it possible to aid the decision makers in forest fighting, by locating the fire front and the places of fire re-ignitions, and by indicating flame heights. Images of a prescribed forest fire obtained in a field campaign have been analyzed to define and validate experimentally the fire index. Comparison with the results of classification by post-processing of multi-spectral images shows a good degree of agreement, demonstrating the effectiveness of the fire-index approach.
\end{abstract}

Keywords: infrared, bi-spectral, forest fires, monitoring.

\section{Introduction}

Infrared (IR) sensors are already commonplace in forest fire related applications [1]. Satellites have become standard for fire danger estimation, mapping of burned areas and follow-up of after-fire recovery, and ground-based platforms are used in many places to provide early fire detection. However, the potentialities of IR for fire monitoring and characterization have not been fully 
realized yet. The very intensity of fire IR emission makes it difficult sometimes to obtain unsaturated images, and most IR cameras are used simply to "see the fire" without a radiometric calibration that would greatly enhance the information they provide.

Moreover, when trying to obtain quantitative measurements, even a calibrated camera faces non-trivial difficulties, which stem from the very nature of forest fires: complex targets that change with time, with several different regions (flames, fire front, embers...) whose spectral emission profiles may be very different from that of a blackbody. Fires, in addition, are usually observed from long distances, and each region, with a different spectral profile, is affected by atmospheric absorption in a different way. Therefore, measured radiances may be quite different from those emitted, and, in particular, apparent temperatures may differ strongly from the real ones, even for a perfectly calibrated camera.

In previous works, a scene analysis was performed using multi-spectral images that made possible to distinguish between fire regions ("classes") [2]. This classification allows to take into account the different spectral emission profiles of the regions and consequently to take full advantage from camera calibration. To obtain physical magnitudes, like embers temperature or radiated power, becomes thus possible [3].

However, this scene analysis is a relatively complex process, which can only be made with a post-processing of the IR sequences of images acquired during the burn. It is very useful for fire studies, but it does not improve fire monitoring. What is needed to help forest fire fighting is a tool that can be operated in real time. To this end, only a schematic scene analysis is necessary, in order to distinguish hot embers from active fire areas (fire front and re-ignition places) and, if possibly, to give an indication of flame height.

In this work we show that this can be done with a medium IR bi-spectral system and an extremely simple processing, thus making it possible to operate in real time. The method is applied to a field test burn of a suppression fire, and is validated by comparison with the results of the full post-processing method applied to a multi-spectral 3-band system.

\section{Classification of IR multi-spectral forest fire images}

The problem of classifying a fire scene, i.e., of assigning each pixel to a meaningful "class" previously determined (for instance, fire front, embers, flames, or background) can be solved along the lines well established in the field of satellite remote sensing, where multi-spectral images are used to make "thematic maps" of land use, assigning each pixel to a specific class (for instance, water, sand, urban areas or specific crops).

In the standard form used in satellite remote sensing, this process involves an algorithm called "classifier". Pixels in an image that are known to correspond to specific regions in the "ground truth" are designated as pure representatives of that class (usually called "endmembers"). For an n-band multispectral system, a 
pixel can be visualized as a point in an n-dimensional "multispectral space". An image translates into an n-dimensional scatterplot: a cloud of points, one for each pixel, where pixels of similar spectral composition are neighbors. Points corresponding to the endmembers are fed to the algorithm in a process that is called "training the classifier" [4]. After training, the classifier runs over all the pixels of the image and assigns each of them to the class whose endmembers are more similar to it, according to some specific criteria. This process is tedious since it generally requires a lot of ground truth images to train the algorithm, in particular when the dimensionality of the data (number of bands) grows.

In previous works [2] we demonstrated that, for fires observed at short distances (a few meters), this method can be simplified by using only two bands in the medium infrared, one at the $\mathrm{CO}_{2}$ emission band near $4.26 \mu \mathrm{m}$ and other outside it. For these bi-spectral images, the multispectral space reduces to a 2dimensional space, and the scatterplot is simply a 2D graph where each axis corresponds to the radiance in one band, and the spectral composition of each pixel can be appreciated visually. Pixels that clearly correspond to specific regions, like embers or flames, can be identified on the scatterplot and selected as the endmembers for that class. The classifier assigns then the rest of the pixels to a class, in our case, by a maximum likelihood criterion.

This method of "classification on the scatterplot" simplifies greatly the process, since no ground truth images are needed. This simplification is possible because of the large contrast in the spectral composition of "flame" and "embers" regions, due to the strong $\mathrm{CO}_{2}$ emission band. However, as distance increases, the atmospheric $\mathrm{CO}_{2}$ absorbs this band, and classification becomes more difficult. "Classification on the scatterplot" techniques applied to direct images don't work well for fires at distances of hundreds of meters [5]. The obvious way out of this difficulty would be to use more bands, but this means, in principle, to use the full process of classifier training. This would be a tiresome work with uncertain results, because fires are not standard targets, and a large array of multispectral images with well-defined endmembers is not available.

Fortunately, classification on the scatterplot can still be feasible by applying a technique called Principal Component Analysis (PCA) to the original multispectral data. This is a standard method of image processing [4] that produces uncorrelated bands by making linear combinations of the often highly correlated original multispectral bands. Each combination is a "principal component" and there are as many PC bands as original bands. The transformation is in fact a translation in multi-spectral space that takes the origin to the center of mass of the cloud of points, followed by a rotation in such a way to obtain a diagonal covariance matrix, i.e., in order to orientate the coordinate axes of the space along the main axes of the cloud. This means that the PC images are uncorrelated. In addition, PCs are ordered by decreasing eigenvalue of the covariance matrix, i.e., PC1 has the larger variance, PC2 the second largest variance, and so on. This means that most of the information is contained in the first PC bands. 
Therefore, when working with multi-spectral images, it may be wise to perform a PCA and retain only the first PC bands. This strategy applied to threeband images of experimental field burns has made possible classification using the PC2 vs. PC1 scatterplot up to at least $550 \mathrm{~m}$ of distance [5].

Although this method is simple as compared to standard classification techniques, it still requires a lot of computational load, since PCs have to be calculated for each frame, and the endmembers have to be identified manually on the scatterplot. Therefore, it cannot be performed in real time. This, in practice, is not an important problem when the aim is to obtain physical parameters like radiated power, since this processing is inherently complex: in addition to classification, images must be geo-referenced and an area must be assigned to each pixel. However, real time operation would be a very convenient feature to aid in forest fire fighting and suppression. A feasible system for real time operation must use as few bands as possible and keep processing extremely simple. In particular:

- Standard classification techniques, which train the classifier with wellknown points in the ground truth, are prohibitively complex.

- PCA should not be used, since the coefficients of the linear combinations making up the PCs must be recalculated at each frame.

- Simple "classification on the scatterplot" methods are not feasible, since they require to draw a scatterplot and to identify clusters for each frame.

The simplest approach is to use only two bands and define a fixed combination of them as a "Fire Index", in a similar spirit to the well-known Normalized Difference Vegetation Index. In the following sections we explore this approach, comparing the results of the full 3-band maximum likelihood classification with those of the simple Fire Index-based processing defined here.

\section{Experimental measurements}

Field measurements have been performed on a prescribed suppression fire on an $82 \mathrm{~m} \times 135 \mathrm{~m}$ scrubland plot. The test was conducted and instrumented by CIFAL-LOURIZAN, within the framework of the Fire Paradox research project.

The fire was monitored from a distance of about $480 \mathrm{~m}$ with several imaging systems (figure 1): a bi-spectral system in the medium (MIR) and thermal (TIR) infrared regions; a high speed MIR camera that allows to combine several integration times to increase dynamic range without saturation, and a MIR multispectral system with a rotating four filter wheel. The fire was registered also with a standard video camera in the visible region as a reference. Figure 2 shows an example of simultaneous visible-MIR images.

In this work we study the IR images acquired with the multispectral MIR system. We only have used tree filters with wavelengths centered at: F2 = 3.7 $\mu \mathrm{m}, \mathrm{F} 3=4.0 \mu \mathrm{m}$ and $\mathrm{F} 4=4.7 \mu \mathrm{m}$, and a width at half maximum of about 


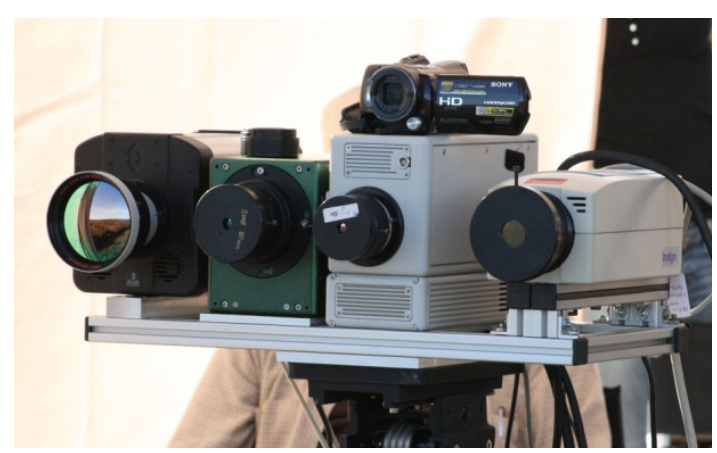

Figure 1: Imaging systems used in the test fire. From left to right, high-speed MIR camera, MIR multispectral system, MIR camera (with visible video camera on top) and TIR camera. The last two cameras integrate a MIR-TIR bi-spectral system.

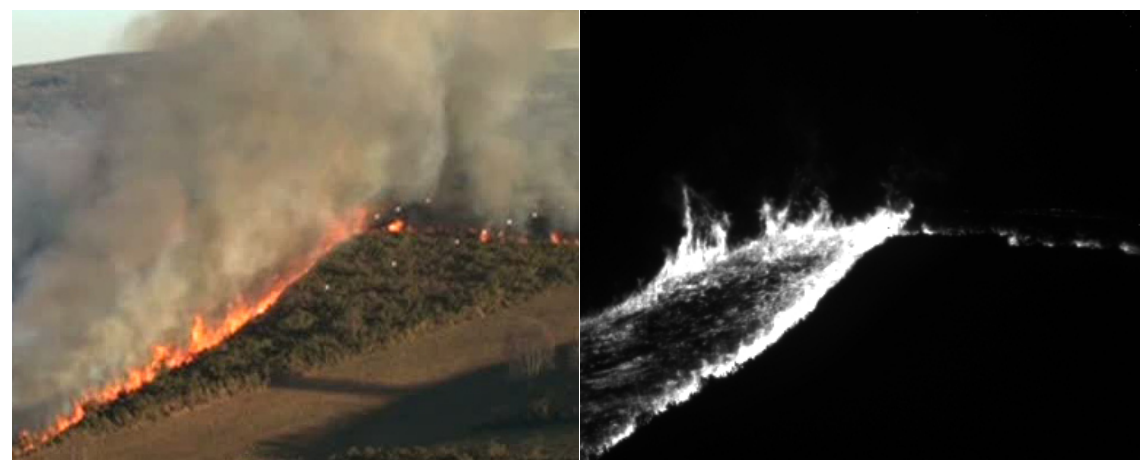

Figure 2: Two simultaneous images of the fire: visible (left) and $4.7 \mu \mathrm{m}$ IR band (right).

$400 \mathrm{~nm}$. Position F1 was reserved to acquire in the entire MIR region, but it was not used to avoid saturation (the high-speed MIR camera images were used instead). The choice of these three filters is motivated by the spectral profile of $\mathrm{CO}_{2}$, the main emitter of combustions in the MIR band. The strong emission band centered at $4.26 \mu \mathrm{m}$ is divided by atmospheric absorption in two spikes: a narrow "blue" spike at short wavelengths and a wide "red" spike at long wavelengths. Filter F4 is centered at the longest wavelength region of the red spike, where atmospheric $\mathrm{CO}_{2}$ absorption is presumably small; F3 filter is centered at the blue spike, and F2 is outside the $\mathrm{CO}_{2}$ band.

\section{Processing}

A sequence of 20 images (one each 5 minutes) of the experimental burn described in the previous section was classified as explained in section 2. For 

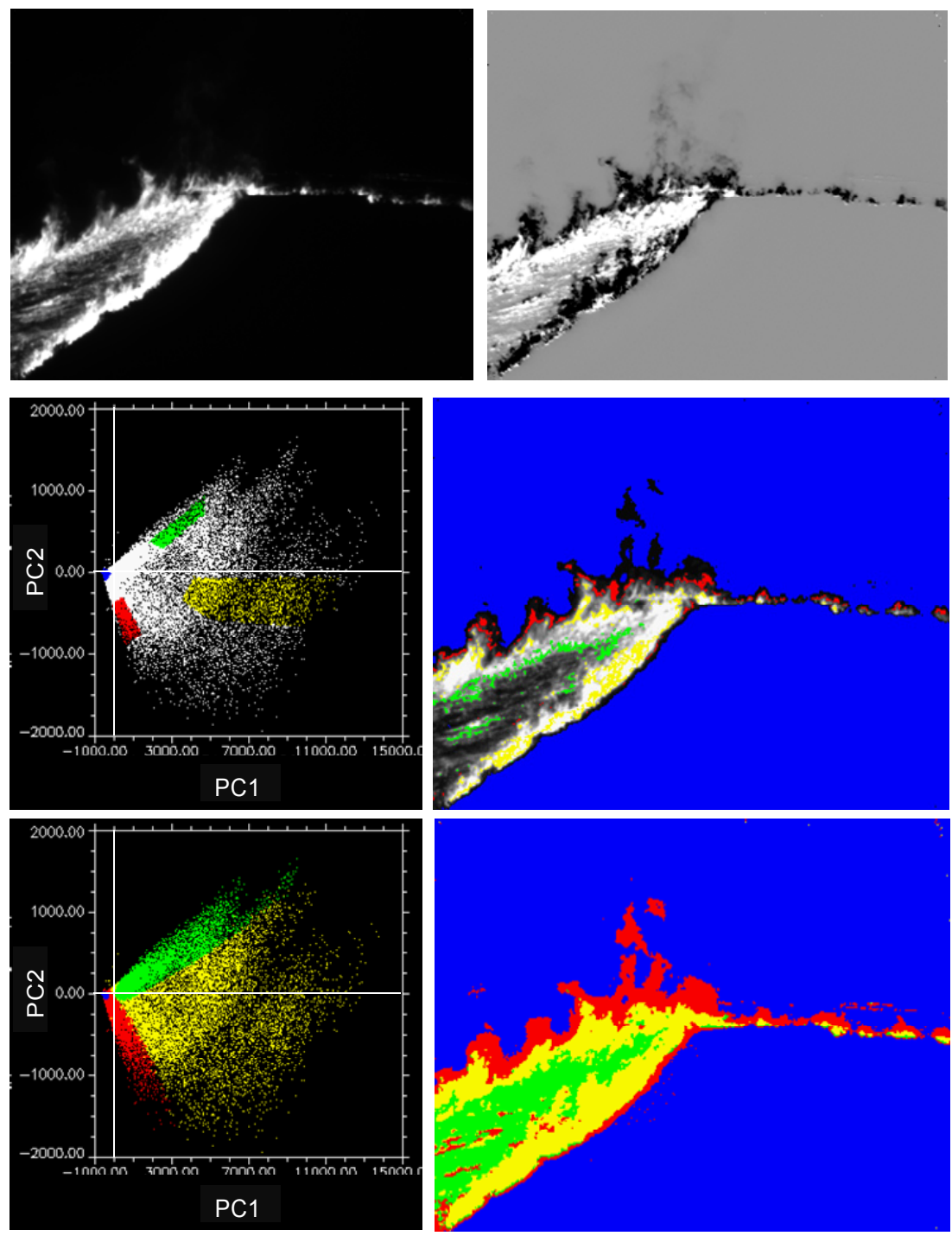

Figure 3: Top row: PC1 (left) and PC2 (right) for a typical frame during the burn. Middle row: PC2 versus PC1 scatterplot of the previous images with endmembers marked (left); position of endmembers on the image (right). Bottom row: Result of the classification on the scatterplot and on the image. The regions are: embers (green), fire front (yellow), flame (red) and background (blue) (color online only). 
each frame, a PC analysis was performed; clusters for the endmembers of the different classes were identified on the PC2 vs. PC1 scatterplot and a maximum likelihood algorithm was used to assign the rest of the pixels to a specific class.

Figure 3 shows this process applied to a specific frame, number 3 . In the top row, the first two principal components are shown. Their expression as combinations of the filters is:

$$
\begin{aligned}
& \mathrm{PC} 1=0.698 * \mathrm{f} 2+0.514 * \mathrm{f} 3+0.498 * \mathrm{f} 4 \\
& \mathrm{PC} 2=0.328 * \mathrm{f} 2+0.389 * \mathrm{f} 3-0.861 * \mathrm{f} 4
\end{aligned}
$$

where $\mathrm{f} 2, \mathrm{f}$, f4 stand for the digital number values of the F2, F3, F4 filters with their respective averages subtracted (so that over the image $\mathrm{f} 2, \mathrm{f} 3$ and $\mathrm{f} 4$ average to zero). Clearly, PC1 (shown at left) is a kind of mean of the three bands, measuring the overall IR brightness. PC2 (shown at right) adds the contributions of F2 and F3 but subtracts, with a larger weight, the contribution of F4. Since this filter collects the contribution of $\mathrm{CO}_{2}$, flames appear with negative values (black in the image).

In the middle row of Figure 3, the PC2 vs. PC1 scatterplot is shown at left, with regions selected as endmembers: green for embers, red for flames, yellow for fire front (flaming embers) and blue for background. The right-hand image shows the location of the endmembers on the image. The result of a classification performed with those endmembers is shown on the bottom row of Figure 2. Each pixel has been assigned to the most similar class, in a maximum likelihood sense; at left it is shown the classified scatterplot; at right, the classified image. This process is repeated for each frame to obtain the classified images. We call this procedure Maximum Likelihood Three-Band (ML3B) classification.

Classification can be simplified if the linear combinations that give the PCs do not change very much as the fire evolves. This is indeed the case, as shown by figure 4 (top row), which shows the weights of PC1 and PC2 on the three filters along the burn. In addition, it is clear that filters F2 $(3.7 \mu \mathrm{m})$ and F3 (4.0 $\mu \mathrm{m})$ play a very similar role in PC1 and PC2. Differences in their value are only important for PC3, but this $\mathrm{PC}$ is not useful for classification.

Therefore, in order to obtain PC1 and PC2 filter F3 is redundant, and it seems plausible to use only filters F2 and F4 to study fires. The PC analysis has been repeated using only these two bands; the resulting PCs have the weights shown on figure 4 (bottom row). Again, weights are very similar along the whole burn. This suggests a way to a very simple processing, using only two bands and two fixed combinations of them given by the nearly constant weights of the PC1 and PC2 calculated for those two bands. In this way we define two "pseudo-PCs" ("pseudo" because they are obtained by a fixed formula, not by an adaptive one as the real PCs):

$$
\begin{aligned}
& \text { psPC1 }=0.813 * \mathrm{f} 2+0.582 * \mathrm{f} 4 \\
& \text { psPC2 }=0.582 * \mathrm{f} 2-0.813 * \mathrm{f} 4
\end{aligned}
$$

To define the weights, we have calculated the PCs of a composite ("mosaic") image formed by all the frames; the average values subtracted from F2 and F4 to obtain $\mathrm{f} 2$ and $\mathrm{f} 4$ have been calculated also for this mosaic image. 

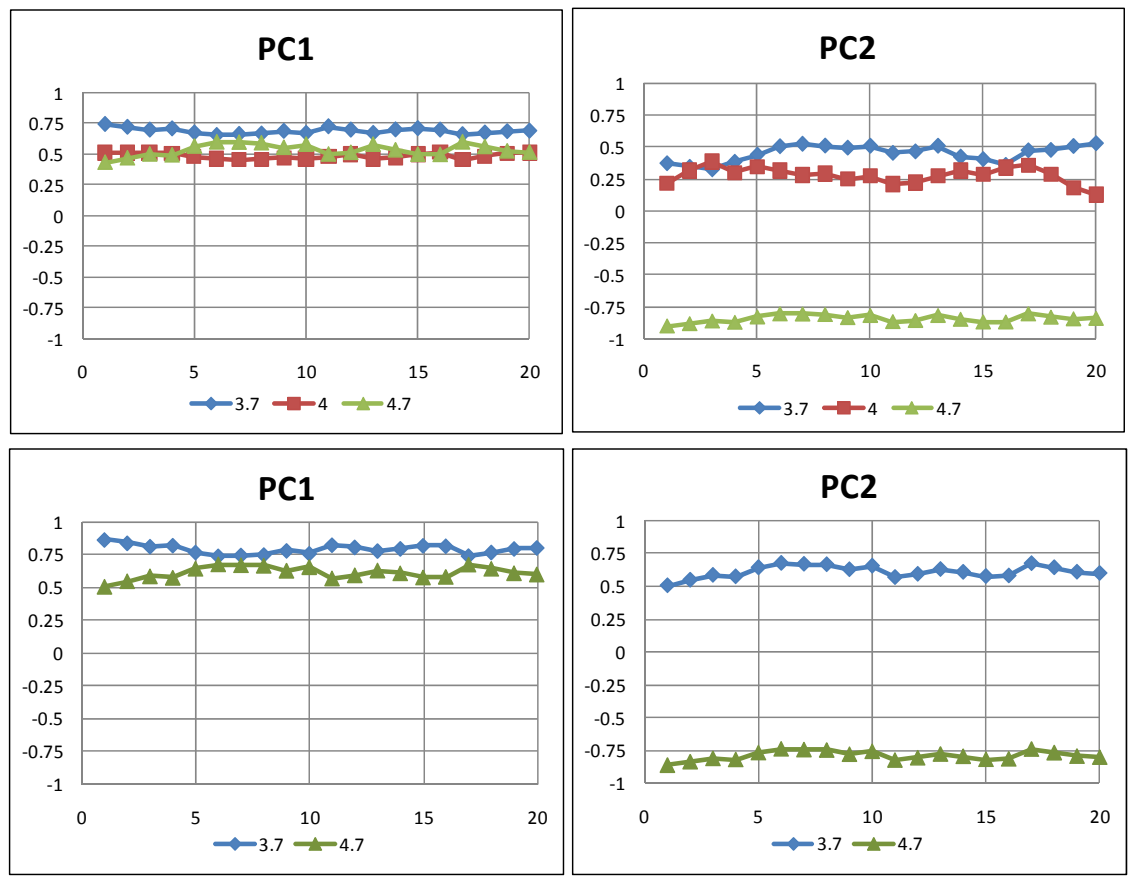

Figure 4: Weights of the two first PCs on the IR bands: Top, for three bands; bottom, for two bands.

These pseudo-PCs should be a good practical approximation of the real PCs for classification purposes. We have compared them graphically in figure 5 . The left side shows the PC2 vs. PC1 scatterplot with the ML3B classes. At the right side, the same classes are shown on the psPC2 vs. psPC1 scatterplot. Although there are some differences on the shape of the scatterplot and the limits between classes are somewhat blurred, the overall pattern is very similar.

It is clear also that frontiers between classes in the scatterplot are quite straight. This suggests the idea of approximating them by straight lines that go through the origin and then to classify pixels according to the value of $\mathrm{psPC} 2 / \mathrm{psPC} 1$. In fact, we will define the ratio $\mathrm{psPC} 2 / \mathrm{psPC} 1$ as the Medium Infrared Fire Index:

$$
\mathrm{MIFI}=\mathrm{psPC} 2 / \mathrm{psPC} 1
$$

If the frontiers between embers and fire front and between fire front and flame are approximated, respectively, by lines with slope $\mathrm{s} 1$ and $\mathrm{s} 2$, then a pixel with psPC1 $>0$ will be assigned to "embers" if MIFI $>\mathrm{s} 1$, to "fire front" if $\mathrm{s} 1>\mathrm{MIFI}$ $>\mathrm{s} 2$, and to "flame" if $\mathrm{s} 2>$ MIFI. If psPC1 $<0$, the pixel is considered as background.

A way to assess this fast "MIFI-classification" is by plotting the histograms of MIFI values for the ML3B classes over the whole fire studied. This has been done 

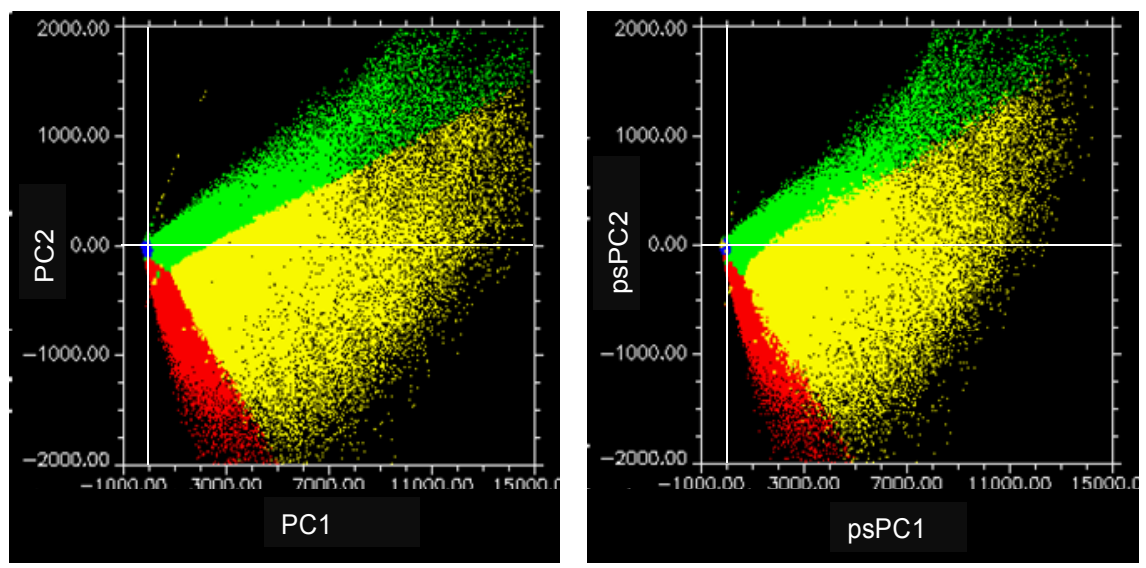

Figure 5: ML3B classes obtained for the whole burn plotted on the PC2 versus PC1 scatterplot (left) and on the psPC2 versus psPC1 scatterplot (right).

in figure 6 (left). Ideally, no overlapping peaks for each class should be obtained. It is clear that there is an important overlap between embers and fire front, but flame is well separated both from embers and fire front. This is in fact a great progress as compared to separability based on PC2 value only, as can be seen in figure 6 (right), where the fire front mixes completely with flame and embers.

Therefore, the MIFI provides a "fast classification" with acceptable results for practical purposes, and, since it applies a simple fixed linear combination to the digital numbers measured by the two F2 $(3.7 \mu \mathrm{m})$ and F4 $(4.7 \mu \mathrm{m})$ channels of a bi-spectral MIR imaging system, it can be implemented for real time operation in the field.

Figure 6 can be used to define the values of the threshold values s1, s2 used to classify the scene. For illustrative purposes we have used s1 $=0.41$, s2 $=0.0$, although these values can be optimized depending on the relative importance of classification errors. An example of the results of this fast classification is provided by figure 7, where the ML3B classes (left) are compared with the MIFI classes (right) for frame number 5, showing a very good agreement.

Results can be summarized in the confusion matrix (table 1), which lists the percentage of pixels of each ML3B that is classified in each of the MIFI classes. Off-diagonal values are classification errors. Although there is an appreciable degree of crossing between the flame, embers and front classes, the overall accuracy is very good: a $96.5 \%$ of pixels correspond to the diagonal of the matrix; i.e., are classified equally by the two methods. If background pixels are excluded, accuracy is still $63.5 \%$, which is an acceptable value taking into account that MIFI-based classification is extremely simple, and that s1, s2 threshold values are not optimized. 


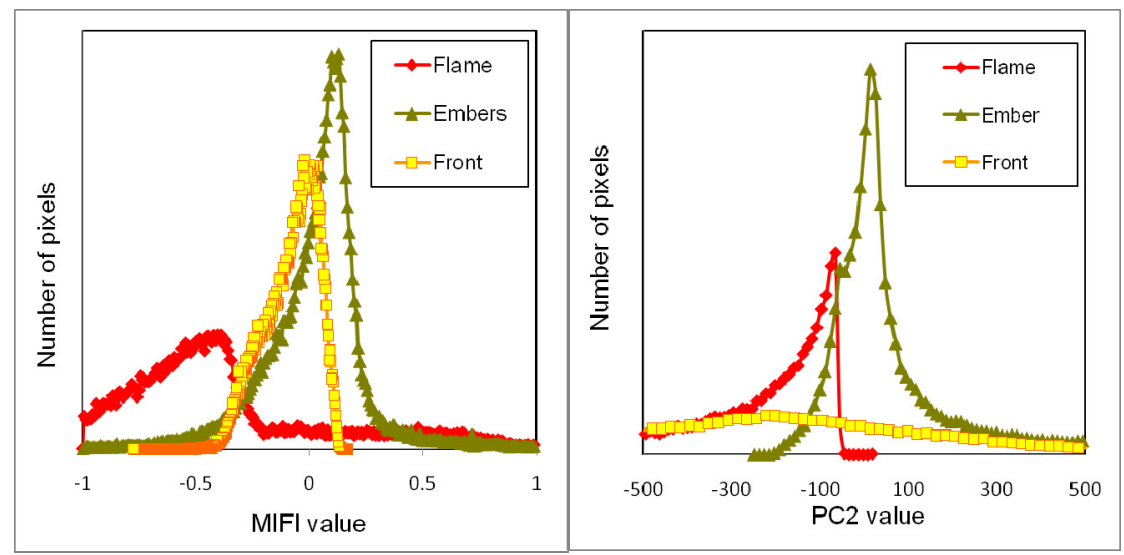

Figure 6: Histograms for the different ML3B classes of: MIFI values (left), PC2 values (right).
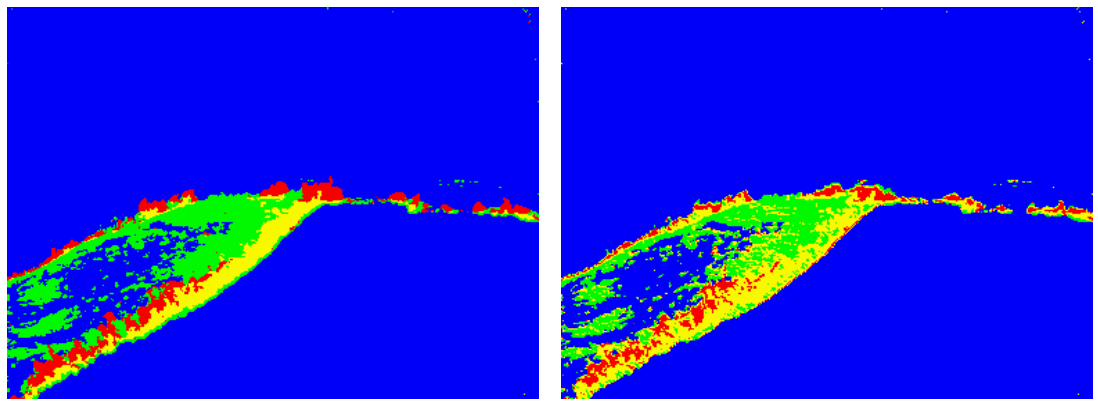

Figure 7: A typical image of the burn classified with ML3B classification (left) and classification based on MIFI values (right).

Table 1: Confusion matrix relating ML3B classification with MIFI classification.

\begin{tabular}{|c|c|c|c|c|c|}
\hline \multirow{2}{*}{$\begin{array}{c}\text { MIFI } \\
\text { Class }\end{array}$} & \multicolumn{4}{|c|}{ ML3B class (Percent) } & \multirow{2}{*}{ Total } \\
\cline { 2 - 6 } & Flame & Embers & Bkg & Front & \\
\hline Flame & 58.69 & 2.98 & 0.15 & 0.23 & 1.63 \\
\hline Embers & 8.23 & 61.41 & 0.16 & 28.25 & 3.28 \\
\hline Bkg & 10.82 & 3.35 & 99.49 & 0.02 & 91.49 \\
\hline Front & 22.26 & 32.26 & 0.2 & 71.5 & 3.6 \\
\hline Total & 100 & 100 & 100 & 100 & 100 \\
\hline
\end{tabular}




\section{Summary and conclusions}

Studies of forest fires in the medium IR have demonstrated that the scene can be classified into different regions using the first two Principal Components (PCs) of multispectral three-band images, at 3.7, 4.0 and $4.7 \mu \mathrm{m}$. Analysis of the spectral weights of these components shows that they change little during the different fire stages, and that filter at $4.0 \mu \mathrm{m}$ is redundant. Thus, pseudo-PCs have been calculated as fixed combinations of digital numbers measured at 3.7 and $4.7 \mu \mathrm{m}$, and the similarity with the real PCs has been assessed. Finally, a Medium Infrared Fire Index (MIFI) has been proposed that is the ratio of the two pseudo PCs: MIFI $=$ psPC2/psPC1. This index is extremely simple in computational terms, so that it can be calculated in real time. The ability of MIFI to perform a fast classification at a distance of $480 \mathrm{~m}$ has been demonstrated by comparing classes obtained from threshold values in MIFI with classes obtained with a maximum likelihood algorithm that uses the real PCs calculated with the three original bands. A global accuracy of $96.5 \%$ has been found that indicates a very good agreement and demonstrates the effectiveness of the fire-index approach.

\section{Acknowledgements}

The authors wish to acknowledge J. A. Vega and E. Jiménez of CIF-Lourizan (Galicia) for the realization of the field tests. Finally, F. Díaz for assistance in the measurements and image processing. This work has been partially funded by Fire Paradox project FP6-018505.

\section{References}

[1] San-Miguel-Ayanz, J. \& Ravail, N., Active Fire Detection for Fire Emergency Management: Potential and Limitations for the Operational Use of Remote Sensing. Natural Hazards, 35, pp. 361-376, 2005.

[2] Aranda, J.M., Meléndez, J., de Castro, A.J., \& López, F., Bi-spectral Infrared Forest Fire Detection and Analysis Using Classification Techniques SPIE Proceedings, 5153, pp. 136-146, 2003.

[3] Meléndez, J., Aranda, J.M., de Castro, A.J., and López, F., Measurement of forest fire parameters with multi-spectral imaging in the medium infrared. QIRT Journal, 3, pp. 183-201, 2006.

[4] J.A. Richards and X. Jia. Remote Sensing Digital Image Analysis, 3rd ed. Springer-Verlag, Berlin, 1999.

[5] Aranda, J. M., Meléndez, J., de Castro, A. J., and López, F., Measurement of physical parameter of forest fires by infrared imaging methods, Proceedings of First International Conference on Modelling, Monitoring and Management of Forest Fire 2008, pp. 111-120, Eds. J. de la Heras, C.a. Brebbia, D. Viegas \& V. Leone. WTT Transactions on Ecology and the Environment, vol. 119 (2008). 